\title{
Integration of BIM work culture for improving global project collaboration productivity
}

\begin{abstract}
Building information modelling is further globalizing architecture, engineering, and construction (AEC) professional partnerships. However, little is known on the effect of cultural and human factors on BIM-enabled visualization applications. This desktop study examined the extant literature on factors relating to application of BIM-enabled visualization technologies as a process that can improve, leverage, and conduct visual communication for coordination during implementation of global projects. It identifies BIM-enabled visualization having the capability in facilitating knowledge flows in complex discontinuous working environment of a property development-s life cycle, and supports designers- understanding in its early working phases. This chapter presents the development of a theoretical proposition for embedding local work culture etiquette in BIM-enabled visualization application for augmenting dynamic knowledge transfer among discontinuous members in a building project. The result is expected to benefit rapidly developing countries (e.g., Malaysia) in enabling successful partnerships with counterparts from developed countries.
\end{abstract}

